С.Р.Джконсон, Дж.Ф.Кордье, К.Лазор, В. Коттен, У.Костабель, С.Харари, М.Рено-Габер, А.Болер, М.Броне, Х.ІІоппер, К.Кингсвуд и рабочая группа по ЛАМ Европейского респираторного общества

Руководство Европейского респираторного

общества по диагностике и лечению лимфангиолейомиоматоза

\author{
S.R.Johnson, J.F.Cordier, R.Lazor, V.Cottin, U.Costabel, S.Harari, M.Reynaud-Gaubert, A.Boehler, M.Brauner, \\ H.Popper, F.Bonetti, C.Kingswood \\ European Respiratory Society guidelines \\ for the diagnosis and management \\ of lymphangioleiomyomatosis
}

Источник: Johnson S.R., Cordier J.F., Lazor R. et al. European Respiratory Society guidelines for the diagnosis and management of lymphangioleiomyomatosis. Eur. Respir. J. 2010; 35: 14-26.

В 2010 г. в Европейском респираторном журнале было опубликовано Руководство по диагностике и лечению лимфангиолейомиоматоза (ЛАМ). Это Руководство было подготовлено группой экспертов по инициативе Европейского респираторного общества (ЕPO).

ЛАМ - редкое заболевание легких, которое может развиться без явной причины или в рамках наследственного заболевания - туберозного склероза (TC) $[1,2]$. Распространенность ЛАМ составляет 1 на 400 тыс. взрослых женщин. Среди лиц с ТС ЛАМ обнаруживают в 30-40 \% случаев [3, 4]. Описаны единичные случаи ЛАМ, ассоциированного с ТС, у мужчин и детей $[5,6]$.

ЛАМ проявляется прогрессирующей одышкой, повторными пневмотораксами, накоплением хилезного выпота в полости плевры и редкими эпизодами кровохарканья [1]. Внелегочная лимфаденопатия и разрастания опухолевых масс, которые принято называть «лимфангиолейомиомами», могут приводить к нарушению циркуляции лимфы в брюшной полости и тазовых органах [7]. У больных ЛАМ часто встречаются ангиолипомы почек [8] и менингиомы [9]. Проявления ЛАМ и темпы прогрессирования значительно варьируются, что затрудняет прогноз заболевания.

Диагноз устанавливают на основании результатов биопсии легких, экстрапульмональных лимфатических узлов и лимфангиолейомиом или сочетания характерных проявлений болезни и картины изменений в легких на компьютерной томографии высокого разрешения (КТВР). Гистологический диагноз основывается на характерной морфологии клеток ЛАМ и результатах иммуногистохимических исследований: положительной реакции на актин гладких мышц и антитела НМВ-45. У пациентов с множественными кистами легких установление диагноза ЛАМ только на основании картины КТВР создает риск диагностических ошибок.
В связи с редкостью ЛАМ контролируемые исследования эффективности лечения заболевания не проводились. Поддерживающая терапия бронхолитиками и кислородом направлена на уменьшение бронхиальной обструкции и гипоксемии. Для устранения пневмо- и хилоторакса и удаления образований в почках используют хирургические методы [10, 11]. Поскольку считается, что ЛАМ стимулируется эстрогенами, в лечении используют овариэктомию, тамоксифен, прогестерон и гонадотропин-рилизинг гормон (GnRH), однако доказательная база эффективности такого лечения отсутствует. Недавнее открытие аномалий генов ТС комплекса 1/2, приводящих к конститутивной активации фермента киназы «мишени рапамицина млекопитающих» (mTOR) [12, 13], послужило обоснованием для применения у пациентов с ЛАМ и ангиомиолипомой ингибиторов mTOR, в т. ч. сиролимуса [14, 15].

Целью группы экспертов ЕРО была разработка основанного на принципах доказательной медицины согласительного руководства по диагностике и лечению ЛАМ. Работу по выработке соглашения проводили 4 комиссии экспертов под руководством 2 председателей при спонсорской поддержке ЕРО.

Процесс выработки руководства проходил поэтапно: 1) постановка вопроса; 2) сбор сведений; 3) оценка доказательности рекомендаций с использованием системы оценки для здравоохранения и науки, разработанной Американской коллегией торакальных врачей в 2004 г. [16]; 4) официальная оценка соглашения и предложений по модификации с использованием статистической шкалы Ликерта [17]; 5) интеграция предложений; 6) последующие повторные оценки соглашения; 7) заключительный пересмотр.

Окончательный вариант рекомендаций учитывал их доказательную силу (от максимальной А до неубедительной I), качество доказательства, пользу, силу согласия экспертов. Полная версия соглашения доступна на сайте: www.ersnet.org/LAMguidelines. 
Предлагаемые определения и диагностические подходы

\section{Диагностические критерии}

Оценка точности диагностики ЛАМ без биопсии легкого не проводилась. Приведенные ниже критерии основываются на результатах нескольких крупных исследований [3, 18-21] и мнении экспертов.

\section{Определенный ЛАМ}

1) характерные а или похожие ${ }^{\text {a }}$ изменения в легких на КТВР и свойственная ЛАМ патологическая картина в биоптате легкого ${ }^{\text {; }}$; или

2) характерные а изменения в легких на КТВР в сочетании с одним из следующих признаков: ангиомиолипомой в почках ${ }^{\mathrm{b}}$, хилезным выпотом в плевральной или брюшной полости ${ }^{\mathrm{c}}$, лимфангиолейомиома ${ }^{\mathrm{d}}$ или поражение ЛАМ лимфатических узлов $^{\mathrm{d}}$, определенный или вероятный ТС ${ }^{\mathrm{e}}$

\section{Вероятный ЛАМ}

1) характерные а изменения в легких на КТВР и похожее ${ }^{\mathrm{f}}$ клиническое течение болезни или

2) похожие а изменения в легких на КТВР в сочетании с одним из следующих признаков: ангиомиолипомой в почках ${ }^{\mathrm{b}}$, хилезным выпотом в плевральной или брюшной полости ${ }^{\mathrm{c}}$.

\section{ВозможныЙ ЛАМ}

Характерные ${ }^{\text {a }}$ или похожие ${ }^{\text {a }}$ изменения в легких на KTBP.

\section{Сноски к вышеизложенному тексту:}

a - согласно приведенному ниже определению;

b - диагноз выставляется по типичным компьютерно-томографическим признакам и / или гистологическому исследованию;

c - на основании визуальных или биохимических характеристик выпота;

$\mathrm{d}$ - на основании гистологического исследования;

е - см. Приложение 2 (в дополнительном материале на сайте: www.erj.ersjournals.com);

$\mathrm{f}$ - похожие клинические признаки включают в себя пневмоторакс (особенно множественный и / или 2-сторонний) и / или нарушения легочной функции, типичные для ЛАМ.

\section{Примечания:}

1) при наличии у пациентов ТС ЛАМ расценивают как ассоциированный с ним (ТС-ЛАМ), в прочих случаях - как спорадический;

2) приведенные выше диагностические критерии ЛАМ применимы только у женщин. У мужчин ТСЛАМ и тем более спорадический ЛАМ встречается крайне редко, поэтому такой диагноз может быть установлен только при типичных или похожих изменениях в легких на КТВР и типичных для ЛАМ гистологических изменениях в биоптате легкого;

3) диагноз ЛАМ требует исключенияя альтернативных заболеваний, характеризующихся кистозны- ми изменениями в легких, особенно при вероятном или возможном ЛАМ (см. дополнительные данные на сайте: www.erj.ersjournals.com, c.18).

Согласие экспертов по диагностическим критериям: очень хорошее.

\section{Гистологические критерии диагноза}

Для ЛАМ типичны 2 вида изменений: кисты и множественная узелковая пролиферация незрелых гладкомышечных клеток и периваскулярно расположенных эпителиоидных клеток - клеток ЛАМ (рис. 1) [22-24]. Оба типа изменений сочетаются в различном соотношении и на ранней стадии заболевания могут быть малозаметными. Чувствительность и специфичность гистологических изменений при ЛАМ не изучались. При появлении типичной пролиферации незрелых гладкомышечных и эпителиоидных клеток вне нормальных мышечных структур, в сочетании с формированием кист, для постановки диагноза в большинстве случаев достаточно рутинной окраски препаратов гематоксилином и эозином наряду с характерными клиническими проявлениями и картиной КТВР.

Иммуногистохимическое выявление актина гладких мышц, десмина и НМВ45 дает ценную дополнительную информацию. Обнаружение НМB45 особенно важно при работе с материалом, полученным путем трансбронхиальной биопсии [25]. В редких случаях реакция на НМВ45 может быть отрицательной, но наличие характерных гистологических признаков дает основание установить диагноз ЛАМ [8, 25]. В подобных случаях корреляция с клиническими проявлениями и КТВР-картиной повышает достоверность диагноза. Примерно в половине случаев иммуногистохимические методы позволяют выявить рецепторы К эстрогенам и / или прогестерону [26, 27].

Дополнительная информация по вопросам диагностики и молекулярной патологии приведена в приложении 3 и доступна на сайте www.erj.ersjournals.com.

\section{Рекомендации}

1. При подозрении на ЛАМ (или другое диффузное паренхиматозное заболевание легких) изучением гистологического материала должен заниматься специалист, имеющий опыт работы с ЛАМ.

2. Предположение о ЛАМ должно возникать при преобладании кист, множественной узелковой пролиферации незрелых гладкомышечных клеток и периваскулярно расположенных эпителиоидных клеток.

3. Иммуногистохимиическая окраска на $\alpha$-актин гладких мышц и НМВ45 должна обязательно выполняться в случаях, когда гистологическая картина не позволяет с уверенностью судить о диагнозе. Иммуногистохимическое выявление рецепторов к эстрогенам и прогестерону помогает в диагностике ЛАМ.

Сила доказательств: мнение экспертов / А. Качество доказательств: мнение экспертов. Польза: значительная. Согласие экспертов: очень хорошее. 

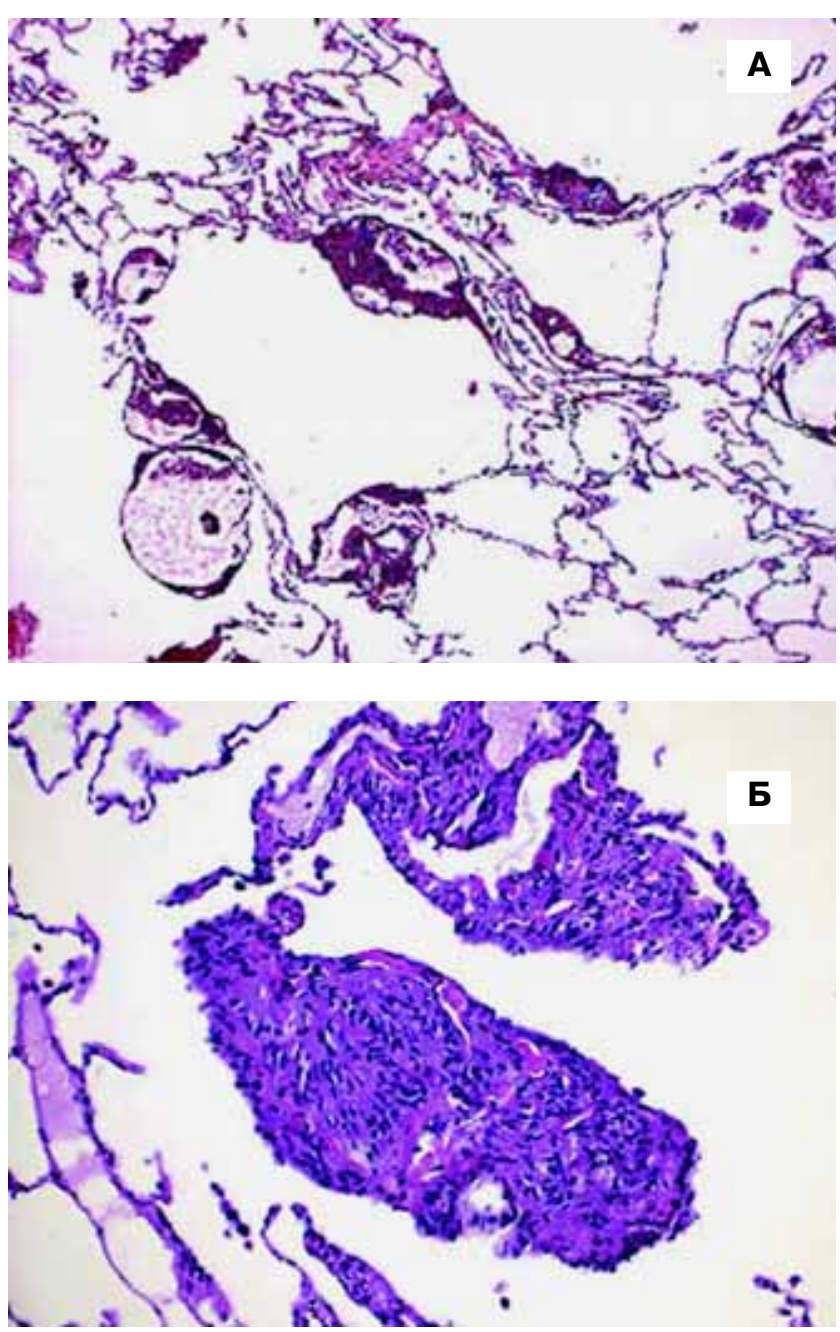

Рис. 1. В биоптате легочной ткани - пролиферирующие скопления ЛАМ-клеток при низком и высоком разрешении (окраска гематоксилином и эозином). Остальные морфологические препараты представлены в дополнении к рис. 1 (www.erj.ersjournals.com).

\section{Рентгенологические критерии диагностики}

\section{Характерные признаки легочного ЛАМ на КТВР}

КТВР рекомендуется как метод лучевой диагностики и для мониторинга изменений при диффузных инфильтративных заболеваниях легких, включая ЛАМ [28]. Кистозные изменения в легких являются главным признаком ЛАМ и обнаруживаются у всех пациентов (рис. 2) [29, 30]. Их внешний вид, размеры и контуры могут значительно варьироваться, обычный диаметр кист - 2-5 мм, но может достигать и 30 мм [31, 32]. Кисты обычно имеют округлую форму и равномерно распределяются в нормальной паренхиме легких. Толщина стенок кист в большинстве случаев варьируется от едва различимой - до 2 мм [32, 33], но может доходить до 4 мм [31].

\section{Рекомендации}

1. Пациентам при подозрении на ЛАМ следует выполнять КТВР легких с использованием тонкого пучка коллимации и алгоритма пространственной реконструкции с высоким разрешением.
2. Исследование может быть выполнено путем последовательного сканирования (среза толщиной 1 мм с интервалом 1 см) или на низкодозовом спиральном мультидетекторном томографе.

Сила доказательств: мнение экспертов / А. Качество доказательств: мнение экспертов. Польза: значительная. Согласие экспертов: очень хорошее.

\section{Замечания}

1. Типичными КТВР-признаками ЛАМ являются множественные (>10) тонкостенные округлые четко очерченные воздушные кисты при нормальном или увеличенном объеме легких и отсутствии других значительных изменений со стороны легких, особенно признаков интерстициального заболевания, за исключением множественной мелкоузелковой гиперплазии альвеолоцитов у пациентов с ТС.

2. КТВР-признаки считаются похожими на ЛАМ, если выявляется всего несколько (от 2 до 10) вышеописанных кист.
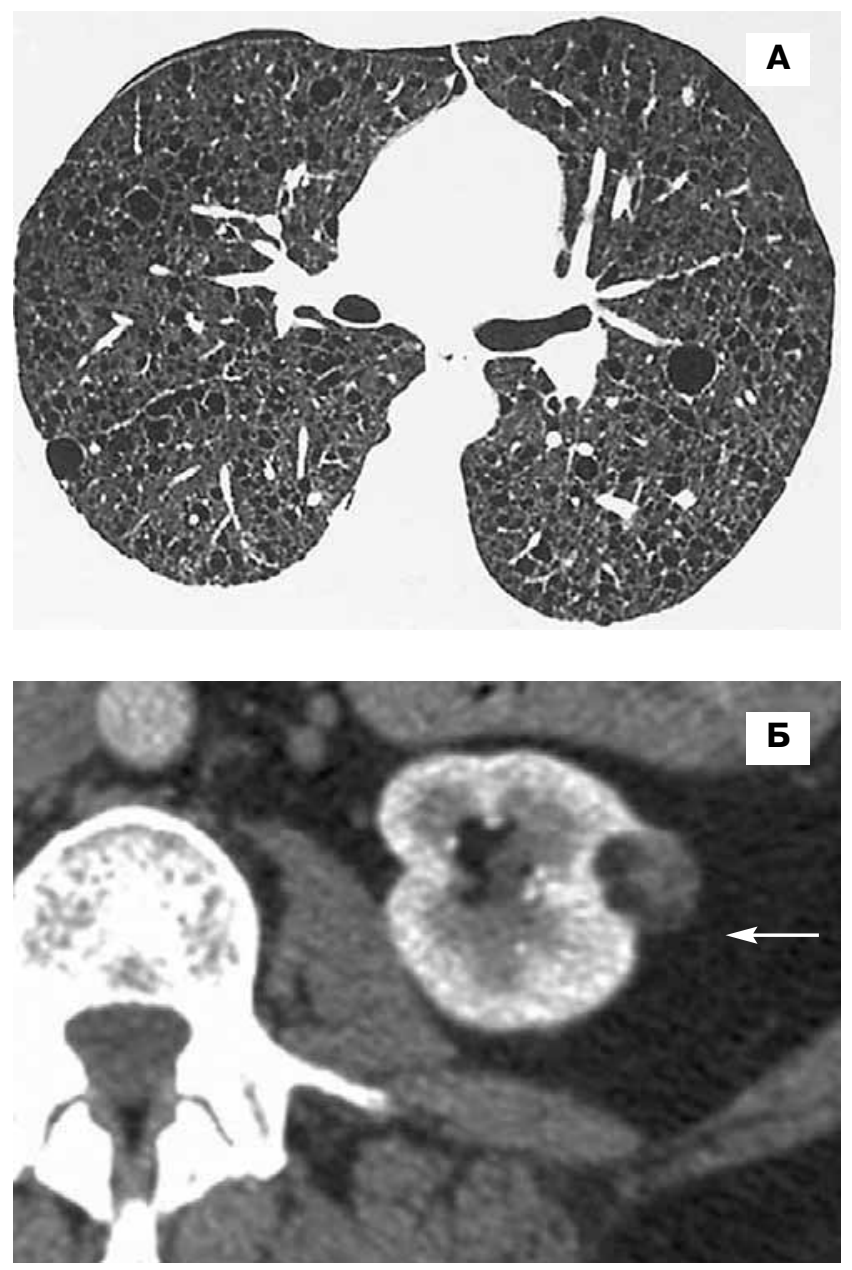

Рис. 2. А - КТВР с типичными изменениями у пациента с умеренно выраженным ЛАМ, осложненным правосторонним пневмотораксом; Б - КТ бессимптомной ангиомиолипомы почки с характерными неоднородными образованиями в левой почке (стрелка). Остальные томограммы легких, почек и брюшной полости представлены в дополнении к рис. 2 (www.erj.ersjournals.com). 
Рентгенодиагностика абдоминальных проявлений ЛАМ

КТ брюшной полости может быть использована с целью выявления ангиомиолипом, лимфангиолейомиом или лимфаденопатии для подтверждения диагноза, определения лечебной тактики и мониторинга динамики изменений. Патологические образования в брюшной полости и тазе обнаруживаются у $2 / 3$ пациентов с ЛАМ [7]. КТ - более чувствительный и специфичный метод, по сравнению с ультразвуковым исследованием, и способен выявить опухоли диаметром < 1 см [7]. В случаях, когда противопоказано применение йодсодержащих контрастных веществ, для выявления жиросодержащих опухолей может быть использована магнитно-резонансная томография (МРТ) с совмещением изображений, полученных с подавлением жировой ткани и без него [34].

\section{Рекомендации}

1. Всем пациентам с ЛАМ или подозрением на ЛАМ следует выполнять КТ органов брюшной полости и таза для выявления ангиолипом и других образований.

2. Исследование брюшной полости нужно проводить в непрерывном режиме с шагом $\leq 3$ мм до и после внутривенного введения неионного контраста.

3. Не рекомендуется обследовать пациентов без клинических проявлений для выявления бессимптомно протекающих лимфангиолейомиом, поскольку на сегодня не существует методов их консервативного лечения.

4. Пациентам с наличием абдоминальных симптомов следует проводить КТ для выявления лимфаденопатии и лимфангиолейомиом.

Сила доказательств: С. Качество доказательств: низкое. Польза: умеренная. Согласие экспертов: очень хорошее.

\section{Выявление менингиом у пациентов со спорадическим ЛАМ}

У пациентов с ЛАМ повышен риск развития менингиом [9], росту которых может способствовать прогестерон. Поиск менингиом особенно необходим у пациентов, получающих прогестерон. Имидж-исследование головного мозга также целесообразно для выявления ТС у пациентов с ЛАМ.

\section{Рекомендации}

1. МРТ головного мозга, выполненная в начале наблюдения, дает информацию для последующих сравнений и должна осуществляться при наличии симптомов, сходных с менингиомой. (Сила доказательств: С. Качество доказательств: низкое. Польза: умеренная. Согласие экспертов: очень хорошее).

2. МРТ головного мозга в качестве скрининга следует проводить женщинам с ЛАМ, получающим прогестативные препараты, или которым планируется их назначить. (Сила доказательств: мнение экспертов / В. Качество доказательств: мнение экспертов. Польза: умеренная. Согласие экспертов: очень хорошее).

\section{Выявление ТС у пациентов с ЛАМ}

У пациентов с явным спорадическим ЛАМ может быть выявлен ТС. Поскольку фенотип ТС очень вариабелен и в $2 / 3$ случаев возникает в результате спонтанных мутаций, заболевание часто не диагностируется. Для исключения ТС необходимы тщательный сбор анамнеза и полное обследование пациентов. В сомнительных случаях требуется консультация клинического генетика. Диагностические критерии ТС и рекомендации по обследованию пациентов приведены в приложении 2 на сайте www.erj.ersjournals.com [35].

\section{Рекомендации}

1. У пациентов с симптомами ЛАМ необходим тщательный сбор как персонального, так и семейного анамнеза, направленный на выявление признаков ТС. Врачебный осмотр должен включать кожные покровы, сетчатку глаза и нервную систему и осуществляться специалистами, знакомыми с проявлениями ТС. (Сила доказательств: В. Качество доказательств: низкое. Польза: значительная. Согласие экспертов: очень хорошее.)

2. Пациентов с ЛАМ и 2-сторонними ангиомиолипомами, а также при наличии сомнений в диагнозе следует направлять к клиническому генетику для полного обследования. (Сила доказательств: D. Качество доказательств: низкое. Польза: значительная. Согласие экспертов: очень хорошее.)

3. Рутинный генетический анализ у пациентов со спорадическим ЛАМ неинформативен и не должен проводиться. (Сила доказательств: D. Качество доказательств: низкое. Польза: отсутствует. Согласие экспертов: очень хорошее.)

\section{Исследование легочной функции}

Показатели объема форсированного выдоха за 1-ю с $\left(\mathrm{OФB}_{1}\right)$ и трансфер-фактора по монооксиду углерода (TLCO) при ЛАМ коррелируют с KT-картиной и гистологическими изменениями в легких и меняются по мере прогрессирования заболевания [4, 30, 36]. Снижение TLCO, выявляемое при ЛАM чаще, чем $\mathrm{OФB}_{1}$, является наиболее чувствительным индикатором ранней стадии заболевания. Кардиореспираторное нагрузочное тестирование (КРНТ) может дать дополнительную информацию, особенно у пациентов с легким течением заболевания, но сложно добиться его удовлетворительной воспроизводимости. Темпы снижения TLCO и OФВ 1 различаются у разных пациентов, поэтому трудно прогнозировать клиническое течение заболевания у конкретного больного и определить, насколько часто следует повторять исследование легочной функции. Большин- 
ство врачей проводят стандартное функциональное исследование каждые 3-6 мес. У пациентов со стабильным течением болезни интервал между исследованиями может быть увеличен до года.

\section{Рекомендации}

1. Спирометрию, бронходилатационный тест и измерение TLCO следует выполнять при первоначальном обследовании пациентов с ЛАМ, включая ЛАМ при ТС. (Сила доказательств: В. Качество доказательств: низкое. Польза: значительная. Согласие экспертов: очень хорошее).

2. Измерение ОФВ 1 и TLCO следует повторять для оценки прогрессирования заболевания и эффективности лечения. (Сила доказательств: В. Качество доказательств: низкое. Польза: значительная. Согласие экспертов: очень хорошее).

3. Исследование функции дыхания следует повторять каждые 3-6 мес. у пациентов с прогрессированием ЛАМ и каждые 6-12 мес. при стабильном состоянии, сохраняющемся в течение 1 года наблюдения. (Сила доказательств: С. Качество доказательств: низкое. Польза: умеренная. Согласие экспертов: очень хорошее).

\section{Исследование газов артериальной крови}

Артериальная гипоксемия при ЛАМ встречается часто [18, 20, 37]. Газовый состав крови при легком и среднетяжелом ЛАМ не несет дополнительной информации к данным пульсоксиметрии. Тем не менее исходные данные могут служить для оценки динамики заболевания, определения показаний для кислородотерапии, оценки функции трансплантата и исключения гиперкапнии на поздних стадиях ЛАМ [38].

\section{Рекомендации}

1. Исследование газов крови может быть выполнено при первоначальном обследовании пациентов с ЛАМ для получения исходных данных и у пациентов с тяжелым заболеванием, в частности, перед трансплантацией легких. (Сила доказательств: мнение экспертов / А. Качество доказательств: мнение экспертов. Польза: значительная. Согласие экспертов: очень хорошее).

2. Исследование газов крови необходимо для определения показаний для кислородотерапии у пациентов с поздними стадиями заболевания. (Сила доказательств: мнение экспертов / А. Качество доказательств: мнение экспертов. Польза: значительная. Согласие экспертов: очень хорошее).

\section{Кардиореспираторное нагрузочное тестирование и тест с 6-минутной ходьбой}

У пациентов с ЛАМ снижены переносимость физической нагрузки и максимальное потребление кислорода $\left(\mathrm{V}^{\prime} \mathrm{O}_{2 \max }\right)$ [39]. Для оценки переносимости физической нагрузки больными ЛАМ можно использовать тест с 6-минутной ходьбой (6-МШТ) [40].

\section{Рекомендации}

1. КРНТ дает дополнительную информацию по сравнению со стандартным исследованием легочной функции у пациентов с клиническими проявлениями ЛАМ. (Сила доказательств: С. Качество доказательств: низкое. Польза: слабая. Согласие экспертов: очень хорошее).

2. 6-МШТ может использоваться для определения степени инвалидизации, прогрессирования болезни и ответа на лечение у пациентов с клиническими проявлениями ЛАМ. (Сила доказательств: мнение экспертов / В. Качество доказательств: мнение экспертов. Польза: умеренная. Согласие экспертов: очень хорошее).

\section{Выявление легочной гипертензии}

Легочная гипертензия не часто наблюдается у пациентов с ЛАМ [39]. Данные об эффективности лечения легочной гипертензии при ЛАМ отсутствуют.

\section{Рекомендации}

1. У пациентов с нетяжелым ЛАМ не рекомендуется скрининговое обследование для выявления легочной гипертензии. (Сила доказательств: неубедительная. Качество доказательств: низкое. Польза: сомнительная. Согласие экспертов: очень хорошее).

2. Измерение давления в легочной артерии при эхокардиографии может проводиться у пациентов с тяжелым заболеванием и больных, нуждающихся в длительной кислородотерапии. Измерение давления в легочной артерии должно выполняться у кандидатов на трансплантацию легких. (Сила доказательств: С. Качество доказательств: низкое. Польза: умеренная. Согласие экспертов: очень хорошее).

\section{Выявление ЛАМ в группах риска}

Длительный период между появлением первых симптомов и установлением диагноза ЛАМ позволяет предполагать, что во многих случаях ЛАМ либо длительно не диагностируется, либо ошибочно расценивается как другое заболевание. Ряд безопасных мер, включающих в себя информирование о симптомах пневмоторакса, отказ от использования эстрогенсодержащих препаратов, вакцинация против вируса гриппа и пневмококка, отказ от курения, и мониторирование состояние пациента для выявления прогрессирования заболевания в самом начале могут быть полезны для пациентов и позволят включать их в клинические исследования.

\section{Показана ли КТ женщинам со спонтанным пневмотораксом?}

Низкая распространенность ЛАМ не дает основания рекомендовать КТ грудной клетки с целью диагностики 
ЛАМ при 1-м эпизоде пневмоторакса. КТ грудной клетки может быть оправдана после 2-го эпизода и должна обязательно выполняться после 3-го и последующих эпизодов пневмоторакса, особенно у некурящих женщин, а также если симптомы заболевания (например, одышка) появились до развития пневмоторакса.

\section{Рекомендации}

1. КТ грудной клетки является рутинным методом обследования при 1-м эпизоде пневмоторакса. (Сила доказательств: I. Качество доказательств: низкое. Польза: сомнительная. Согласие экспертов: хорошее).

2. КТ грудной клетки показана для диагностики ЛАМ при клиническом подозрении и отсутствии явных признаков на стандартной рентгенограмме легких. (Сила доказательств: С. Качество доказательств: низкое. Польза: слабая. Согласие экспертов: хорошее).

3. Комиссия не достигла согласия по целесообразности проведения КТ у пациентов с повторными пневмотораксами, сохраняющейся негерметичностью легкого или перед планируемыми хирургическими вмешательствами. (Сила доказательств: I. Качество доказательств: низкое. Польза: сомнительная. Согласие экспертов: отсутствует).

\section{Нужна ли скрининговая КТВР для выявления ЛАМ у женщин с ТС?}

Среди всех женщин с ТС у 26-39 \% имеются кистозные изменения легких, подобные ЛАМ [2, 41, 42]. У большинства из них отсутствуют симптомы легочного заболевания, и течение ЛАМ, диагностированного при скрининге больных с ТС, неизвестно [43]. Поскольку врачебный осмотр, рентгенография грудной клетки и исследование функции дыхания могут не выявить патологии при ЛАМ, КТВР является методом выбора для выявления ЛАМ у этой категории пациентов.

\section{Рекомендации}

1 Женщинам с ТС показано проведение скрининговой КТВР грудной клетки в возрасте 18 лет и, при отсутствии патологических изменений, повторно в возрасте 30-40 лет. (Сила доказательств: С. Качество доказательств: низкое. Польза: умеренная. Согласие экспертов: очень хорошее).

2 КТВР следует повторить при появлении стойких респираторных симптомов. (Сила доказательств: С. Качество доказательств: низкое. Польза: умеренная. Согласие экспертов: очень хорошее).

3 Женщинам с ТС КТВР грудной клетки показана при появлении респираторных симптомов неясного генеза. (Сила доказательств: В. Качество доказательств: низкое. Польза: значительная. Согласие экспертов: очень хорошее).

\section{Следует ли мужчинам с ТС проводить скрининговую КТВР для выявления ЛАМ?}

ЛАМ может возникнуть у мужчин с ТС, но крайне редко $[5,42,44]$. Поскольку мужчины обычно не получают лечения эстрогенсодержащими препаратами, ранняя диагностика ЛАМ у них не имеет преимуществ.

\section{Рекомендации}

1. Мужчины с ТС и респираторными симптомами неясного генеза нуждаются в обследовании соответственно имеющимся симптомам, в т. ч. с использованием КТВР грудной клетки. (Сила доказательств: С. Качество доказательств: низкое. Польза: умеренная. Согласие экспертов: очень хорошеe).

2. Мужчинам, не имеющим респираторных симптомов, скрининговая КТВР для выявления ЛАМ не показана. (Сила доказательств: D. Качество доказательств: низкое. Польза: умеренная. Согласие экспертов: очень хорошее).

\section{Нужен ли скрининг для выявления ЛАМ у женщин со спорадической ангиомиолипомой?}

Частота выявления ангиомиолипом при скрининговой КТ при спорадическом ЛАМ составляет 40-50 \%, при ТС-ЛАМ - около 80 \% [7, 45-47]. У больных ТС ангиомиолипомы крупнее, чаще бывают 2-сторонними и более предрасположены к кровотечению [4-52].

\section{Рекомендации}

1. Скрининговую КТ для выявления ЛАМ можно выполнять пациентам с 1-сторонней ангиомиолипомой, отсутствием клинических признаков ТС и легочных симптомов.

2. Скрининговое обследование для диагностики ТС должно проводиться пациентам с 2-сторонними ангиомиолипомами, и в случае его выявления необходимо дальнейшее обследование для выявления ЛАМ. (Сила доказательств: В. Качество доказательств: низкое. Польза: значительная. Согласие экспертов: очень хорошее).

\section{Прогноз}

\section{Определение прогноза}

Определение прогноза у конкретных пациентов представляет сложную задачу. Степень выраженности гистологических изменений в легких [20, 23] и некоторых показателей легочной функции [20, 36, 53] при установлении диагноза могут прогнозировать выживание или более быстрое снижение легочной функции. ЛАМ при ТС обычно протекает менее тяжело, чем спорадический ЛАМ, хотя это утверждение может быть ошибочным [18, 54]. Ни один из прогностических факторов не оценивался проспек- 
тивно, более того, некоторые из этих показателей всего лишь отражают позднюю стадию заболевания в момент постановки диагноза, что и приводит к ранней летальности. Вероятно, лучшими прогностическими факторами прогрессирования болезни и выживаемости сегодня являются TLCO и ОФВ У больных ТС-ЛАМ более спокойное течение болезни, чем при спорадическом ЛАМ.

\section{Рекомендации}

1. Биопсия легкого не дает прогностической информации и не должна выполняться только с этой целью. (Сила доказательств: D. Качество доказательств: низкое. Польза: отсутствует. Согласие экспертов: очень хорошее).

2. Темпы прогрессирования заболевания могут быть оценены при повторных исследованиях легочной функции через каждые 3-6 мес. в течение 1-го года после уставления диагноза и затем через каждые 3-12 мес. в зависимости от тяжести заболевания и скорости его прогрессирования. (Сила доказательств: С. Качество доказательств: низкое. Польза: умеренная. Согласие экспертов: очень хорошее).

\section{Наблюдение пациентов с ТС-ЛАМ без респираторных симптомов}

Спорадический ЛАМ, как правило, является прогрессирующим заболеванием со снижением легочной функции [4, 21].

Пациентам с прогрессирующим течением ТСЛАМ рекомендуются регулярное наблюдение и повторные исследования функции дыхания для своевременного выявления изменений, требующих вмешательства. У пациентов с ТС-ЛАМ и минимально выраженными симптомами риск тяжелого ЛАМ ниже, чем при спорадическом заболевании [18].

\section{Рекомендации}

1. Пациенты с ТС-ЛАМ и изначально нормальной функцией легких, сохраняющие стабильное состояние в течение 1-го года наблюдения, не нуждаются в регулярном наблюдении пульмонологом и в мониторинге легочной функции. Необходимость в регулярном наблюдении пульмонологом и повторных исследованиях легочной функции возникает при развитии респираторных симптомов. (Сила доказательств: мнение экспертов / С. Качество доказательств: мнение экспертов. Польза: слабая. Согласие экспертов: очень хорошее).

2. Врачи, участвующие в лечении пациентов с ТС, и сами пациенты должны быть информированы о необходимости обращения к пульмонологу при появлении респираторных симптомов. Это положение должно быть отражено в памятке для пациентов и врачей общей практики. (Сила доказательств: мнение экспертов / С. Качество доказательств: мнение экспертов. Польза: слабая. Согласие экспертов: очень хорошее).

\section{Ведение больных}

\section{Общие рекомендации}

Пациентам с ЛАМ, как и с другими заболеваниями легких, следует напоминать о необходимости поддержания нормальной массы тела и воздержания от курения. Диагноз редкого заболевания и его последствия часто вызывают у пациентов ощущение изоляции. Объединение пациентов в группы помогает решить многие вопросы.

\section{Советы пациентам с пневмотораксом}

При ЛАМ повышается риск развития пневмоторакca, который возникает примерно у $40 \%$ пациентов как первое проявление заболевания и в $66 \%$ случаев после установления диагноза [10, 55]. Частота повторных пневмотораксов при ЛАМ составляет около $75 \%$ [29].

\section{Рекомендации}

Пациенты как со спорадическим ЛАМ, так и с ТСЛАМ, включая больных с минимально выраженными клиническими симптомами или их отсутствием, должны быть предупреждены о риске возникновения пневмоторакса и о необходимости срочно обратиться за медицинской помощью при появлении признаков пневмоторакса.

Сила доказательств: А. Качество доказательств: среднее. Польза: значительная. Согласие экспертов: очень хорошее.

\section{Советы женщинам с ЛАМ и ведение беременности}

Вероятно, что беременность при ЛАМ создает повышенный риск развития пневмоторакса и хилоторакса [10, 56-60] и может ускорять снижение функции легких [61]. Возможно, что женщины с изначально низкими показателями легочной функции хуже переносят пневмоторакс и хилоторакс во время беременности. Беременность также повышает риск кровотечения из ангиомиолипом [62-65].

\section{Рекомендации}

1. Решение о беременности принимает сама женщина. Однако все женщины с ЛАМ, включая тех, у кого симптомы отсутствуют или выражены минимально, должны быть проинформированы о повышенном риске развития пневмоторакса и хилоторакса в период беременности. Этот риск еще более повышается у женщин, перенесших повторные пневмотораксы или плевральный выпот вне беременности, а также у женщин с низкими показателями легочной функции. (Сила доказательств: мнение экспертов / В. Качество доказательств: мнение экспертов. Польза: умеренная. Согласие экспертов: очень хорошее).

2. Беременные женщины с ЛАМ до или вскоре после наступления беременности должны быть проинформированы о повышенном риске развития 
пневмоторакса, хилоторакса и кровотечения из ангиомиолипом. (Сила доказательств: мнение экспертов / В. Качество доказательств: мнение экспертов. Польза: умеренная. Согласие экспертов: очень хорошее).

3. Женщины с ТС перед наступлением беременности должны получить консультацию у генетика. (Сила доказательств: А. Качество доказательств: хорошее. Польза: значительная. Согласие экспертов: очень хорошее).

4. Во время беременности женщины нуждаются в наблюдении пульмонологом и гинекологом, знакомыми с особенностями ЛАМ. (Сила доказательств: мнение экспертов / В. Качество доказательств: мнение экспертов. Польза: умеренная. Согласие экспертов: очень хорошее).

5. Представляется целесообразным разубедить женщин с тяжелым заболеванием в решении забеременеть, но это решение должно быть индивидуальным. (Сила доказательств: мнение экспертов / В. Качество доказательств: мнение экспертов. Польза: умеренная. Согласие экспертов: очень хорошее).

\section{Отказ от эстрогенов, включая контрацептивы и гормонзаместительную терапию (ГЗТ)}

Прием эстрогенов может вызвать прогрессирование легочного ЛАМ [66-69].

\section{Рекомендации}

Женщины с ЛАМ должны избегать приема эстрогенсодержащих препаратов, включая комбинированные пероральные контрацептивы и ГЗТ. (Сила доказательств: С. Качество доказательств: низкое. Польза: умеренная. Согласие экспертов: очень хорошее).

\section{Информация для пациентов о воздушных перелетах}

Сообщения о развитии пневмоторакса во время воздушных перелетов дают основание для совета больным ЛАМ не путешествовать по воздуху [70]. Больные спорадическим и ТС-ЛАМ при сохранной функции легких не нуждаются в особых мерах предосторожности или отказе от воздушных перелетов. Пациентам с поздними стадиями заболевания следует обсудить возможность ингаляций кислорода во время перелета для профилактики гипоксемии и осложнений пневмоторакса в случае его развития.

\section{Рекомендации}

1. Пациентам со спорадическим ЛАМ или ТС-ЛАМ при минимально выраженных симптомах воздушные перелеты не противопоказаны. Их следует предупредить о необходимости отказа от перелетов при появлении новых респираторных симптомов до консультации со специалистом. (Сила доказательств: С. Качество доказательств: низкое. Польза: умеренная. Согласие экспертов: очень хорошее).
2. Пациенты с поздними стадиями заболевания должны обсудить необходимость кислородной поддержки во время перелета и воздерживаться от перелетов при появлении новых респираторных симптомов до консультации со специалистом. Если нелеченный пневмоторакс у таких больных может иметь серьезные последствия, следует искать альтернативные способы путешествия. (Сила доказательств: С. Качество доказательств: низкое. Польза: умеренная. Согласие экспертов: очень хорошее).

3. Авиаперелеты противопоказаны пациентам с диагностированным, но нелеченным пневмотораксом или перенесшим пневмоторакс в течение предыдущего месяца. (Сила доказательств: В. Качество доказательств: низкое. Польза: значительная. Согласие экспертов: очень хорошее).

\section{Легочная реабилитация}

Специальные исследования роли легочной реабилитации при ЛАМ не проводились, тем не менее на больных ЛАМ можно экстраполировать результаты, полученные при других заболеваниях легких, в т. ч. ХОБЛ [71].

\section{Рекомендации}

Легочная реабилитация должна рекомендоваться пациентам с ЛАМ, страдающим от одышки. (Сила доказательств: мнение экспертов / В. Качество доказательств: мнение экспертов. Польза: умеренная. Согласие экспертов: очень хорошее).

\section{Вакцинация против гриппа и пневмококка}

Эффект профилактической вакцинации больных с ЛАМ не изучался, но, по аналогии с ХОБЛ, ее можно рекомендовать пациентам со сниженной функцией легких [72].

\section{Рекомендации}

Пациентам с ЛАМ следует предлагать вакцинацию против гриппа и пневмококка. (Сила доказательств: мнение экспертов / В. Качество доказательств: мнение экспертов. Польза: умеренная. Согласие экспертов: очень хорошее).

\section{Диагностика и лечение остеопороза}

У значительной части пациентов с ЛАМ снижена минеральная плотность костей [73]. Учитывая быстрое снижение минеральной плотности костей после трансплантации легких, пациентам с тяжелым легочным ЛАМ и остеопенией любой локализации рекомендуется раннее начало агрессивной терапии. Также желательны силовые тренировки.

\section{Рекомендации}

1. У пациентов с ЛАМ, особенно у женщин в постменопаузе, следует периодически исследовать минеральную плотность костей. (Сила доказа- 
тельств: В. Качество доказательств: низкое. Польза: значительная. Согласие экспертов: очень хорошее).

2. При выявлении остеопороза у больных ЛАМ его следует лечить как остеопороз при других заболеваниях. (Сила доказательств: В. Качество доказательств: низкое. Польза: значительная. Согласие экспертов: очень хорошее).

3. Учитывая быстрое снижение минеральной плотности костей после трансплантации легких, пациентам с тяжелым легочным ЛАМ и остеопенией любой локализации рекомендуется раннее начало агрессивной терапии. Помимо лекарственного лечения, рекомендуются силовые тренировки в связи с растущим объемом доказательств, что силовые тренировки улучшают костную плотность. (Сила доказательств: В. Качество доказательств: низкое. Польза: значительная. Согласие экспертов: очень хорошее).

\section{Ингаляционные бронходилататоры}

Четверть пациентов с ЛАМ отвечают на ингаляционные бронходилататоры по стандартным объективным критериям, еще у большей части может быть достигнут клинический эффект $[8,36]$. У пациентов, отвечающих на бронходилататоры, обычно имеется бронхиальная обструкция и ОФВ 1 снижается быстрее. У некоторых пациентов с ЛАМ имеются воспалительные изменения в бронхиолах [36], тем не менее эффективность ингаляционных кортикостероидов при этом заболевании не изучалась.

\section{Рекомендации}

Пациентам с бронхиальной обструкцией целесообразно провести пробное лечение бронходилататорами и при их эффективности продолжить лечение. (Сила доказательств: В. Качество доказательств: низкое. Польза: значительная. Согласие экспертов: очень хорошее).

\section{Гормональная терапия: прогестерон}

Несмотря на широкое использование прогестерона при ЛАМ [18], радомизированные плацебо-контролируемые исследования его эффективности не проводились. Результаты некоторых серий случаев и отдельных наблюдений позволяют предполагать, что у некоторых пациентов такое лечение эффективно. Тем не менее серии случаев не позволяют сделать заключение о целесообразности назначения прогестерона, поскольку сообщения об успешном лечении публикуются значительно чаще, чем о неэффективном.

Прогестерон чаще назначался пациентам с более быстрым прогрессированием заболевания. Применение прогестерона у таких больных было, как правило, неэффективным с точки зрения ухудшения легочной функции или одышки и только в 1 наблюдении было получено незначительное снижение темпов падения TLCO [4, 21].

\section{Рекомендации}

1. Прогестерон (перорально или внутримышечно) не следует широко применять у больных ЛАМ. (Сила доказательств: І. Качество доказательств: низкое. Польза: сомнительная. Согласие экспертов: очень хорошее).

2. У больных с быстрым прогрессированием легочной функции или респираторных симптомов может назначаться пробное лечение внутримышечным прогестероном. (Сила доказательств: С. Качество доказательств: низкое. Польза: слабая. Согласие экспертов: очень хорошее).

3. Лечение прогестероном следует продолжать 12 мес. с оценкой клинического состояния и легочной функции каждые 3 мес. Если спустя 1 год лечения выраженность симптомов и функция легких продолжают ухудшаться с той же скоростью, лечение следует прекратить. (Сила доказательств: мнение экспертов / С. Качество доказательств: мнение экспертов. Польза: слабая. Согласие экспертов: очень хорошее).

\section{Гормональная терапия: прочие антиэстрогенные препараты}

Несмотря на многочисленные сообщения о применении овариэктомии [74-78], тамоксифена [79-82] и агонистов GnRH [83-85] при ЛАМ, эффективность этой антиэстрогенной стратегии не подтверждена убедительными доказательствами. Результаты 1 недавно проведенного открытого исследования, в котором 11 пациентов лечились агонистом GnRH трипторелином, показали полное отсутствие эффекта и снижение минеральной плотности костей на фоне лечения [86].

\section{Рекомендации}

Пациентам с ЛАМ не показано какое-либо гормональное лечение, кроме прогестерона. (Сила доказательств: І. Качество доказательств: низкое. Польза: сомнительная. Согласие экспертов: очень хорошее).

\section{Ингибиторы ITOR}

Наследуемые мутации генов TSC-1 и TSC-2 вызывают ТС, в то время как приобретенные (соматические) мутации любого из этих генов ассоциируются со спорадическим ЛАМ. При ЛАМ происходит конститутивная активация m-TOR $[12,13]$. В 2 проспективных открытых клинических исследованиях было показано уменьшение объема ангиомиолипом под влиянием ингибитора m-TOR сиролимуса [14, 15], но при этом не оценивалось влияние препарата на кровотечение из ангиомиолипом. Более того, не проводилось сравнения соотношения риск / польза с другими видами лечения ангиомиолипом (катетерной эмболизацией и консервативной хирургией). Влияние ингибиторов m-TOR на функцию легких не изучено; эти препараты могут вызывать побочные 
эффекты. В будущем ингибиторы m-TOR могут найти применение в лечении ЛАМ, но требуются дальнейшие исследования.

\section{Рекомендации}

1. Сиролимус не следует применять для лечения ЛАМ вне клинических исследований. Больные ЛАМ должны принимать участие в клинических испытаниях при любой возможности. (Сила доказательств: С. Качество доказательств: низкое. Польза: слабая. Согласие экспертов: очень хорошеe).

2. Ингибиторы m-TOR не являются препаратами первого выбора при ангиомиолипомах почек. Сиролимус можно применять в специализированных клиниках у отдельных пациентов с клинически значимыми ангиомиолипомами или лимфангиолейомиоматозными узлами, не подлежащими эмболизации или консервативной хирургии. (Сила доказательств: С. Качество доказательств: низкое. Польза: слабая. Согласие экспертов: очень хорошее).

3. Учитывая вероятную эффективность лечения сиролимусом при отсутствии научных доказательств, возможно назначение препарата отдельным пациентам с быстрым ухудшением функции легких или нарастанием симптомов в специализированных центрах после тщательной оценки соотношения риска и пользы. В период лечения сиролимусом необходимо каждые 3 мес. тщательно оценивать эффекты лечения, переносимость препарата и влияние на функцию легких. При включении пациента в лист ожидания трансплантации легких лечение сиролимусом необходимо прекратить. (Сила доказательств: С. Качество доказательств: низкое. Польза: слабая. Согласие экспертов: очень хорошее).

\section{Осложнения и сопутствующие заболевания}

\section{Ведение больных с пневмотораксом}

Пневмоторакс, возникающий у большинства пациентов, приводит к длительным госпитализациям и часто рецидивирует. Консервативное лечение связано с более высокой частотой рецидивов, чем плевродез через дренажную трубку или соответствующие хирургические вмешательства $[10,15]$. Трансплантация легких у пациентов, ранее подвергавшихся хирургическим вмешательствам на легких или плевре, связана с техническими сложностями и высоким риском периоперационного кровотечения [38, 55, 87, 88].

\section{Рекомендации}

1. Лечение пневмоторакса при ЛАМ должно проводиться совместно пульмонологом и торакальным хирургом. (Сила доказательств: С. Качество доказательств: низкое. Польза: умеренная. Согласие экспертов: очень хорошее).
2. При 1-м эпизоде пневмоторакса можно провести химический плевродез. При неэффективности первоначального лечения, включая плевродез, показано хирургическое вмешательство с учетом клинического состояния больного и опыта местных врачей. (Сила доказательств: С. Качество доказательств: низкое. Польза: умеренная. Согласие экспертов: очень хорошее).

3. Пациентам с повторным пневмотораксом показано хирургическое вмешательство с учетом их клинического состояния и опыта местных врачей. (Сила доказательств: С. Качество доказательств: низкое. Польза: умеренная. Согласие экспертов: очень хорошее).

4. Пациентам, перенесшим плевродез или плеврэктомию, трансплантацию легких во избежание плевральных осложнений нужно производить в трансплантационных центрах, имеющих опыт работы с ЛАМ. (Сила доказательств: С. Качество доказательств: низкое. Польза: умеренная. Согласие экспертов: очень хорошее).

5. Ранее перенесенные плевродез или плеврэктомия у пациентов с ЛАМ не являются противопоказанием для трансплантации легких. Однако такие пациенты должны быть информированы о повышенном риске периоперационного плеврального кровотечения. (Сила доказательств: С. Качество доказательств: низкое. Польза: умеренная. Согласие экспертов: очень хорошее).

\section{Лечение хилоторакса}

Хилоторакс при ЛАМ может протекать почти бессимптомно, но может вызывать выраженную одышку. Методы лечения хилоторакса при ЛАМ [11, 37, 81, 89-94] выбирают с учетом объема и клинической значимости выпота, сопутствующих заболеваний и местного опыта [91]. Для уменьшения выработки хилезной жидкости пациентам иногда назначают безжировую диету (с добавлением пероральных триглицеридов среднего размера или без) или безжировое парентеральное питание [95]. При небольшом объеме выпота показаны наблюдение или торакоцентез.

\section{Рекомендации}

1. Пациентам с хилотораксом назначают безжировую диету с добавлением среднецепочечных триглицеридов. (Сила доказательств: мнение экспертов / С. Качество доказательств: мнение экспертов. Польза: слабая. Согласие экспертов: очень хорошее).

2. Лечение клинически значимого хилоторакса выбирают индивидуально с учетом клинического состояния больного, объема выпота, склонности к рецидивированию, респираторного статуса и возможности трансплантации легких в будущем. (Сила доказательств: мнение экспертов / В. Качество доказательств: мнение экспертов. Польза: умеренная. Согласие экспертов: очень хорошее). 


\section{Лечение и наблюдение больных с ангиомиолипомами}

Опыт основывается на описаниях серий случаев. Эмболизация и нефрон-сберегающие хирургические вмешательства достаточно безопасны, не оказывают негативного влияния на функцию почек как у специально отобранных больных [96, 97], так и при острых почечных кровотечениях [98], в т. ч. у беременных [64]. Эмболизацию можно выполнять при остром кровотечении, она менее инвазивна, не требует общей анестезии, но иногда ее приходится проводить повторно. У пациентов с кровотечением из ангиомиолипомы эмболизация более предпочтительна, чем хирургическое вмешательство, хотя исследования, сравнивающие эти 2 метода, не проводились. Выбор вмешательства определяется техническими факторами, связанными с опухолью, и местным опытом. При отсутствии кровотечения предпочтительны нефрон-сберегающие хирургические вмешательства, особенно при подозрении на злокачественное образование. Необходимо помнить о риске ложной диагностики карциномы [99]. В сомнительных случаях интраоперационная экспрессбиопсия с исследованием замороженных срезов помогает принять решение относительно объема операции. Лучше всего принимать решение индивидуально после скринингового выявления ангиомиолипом или в зависимости от клинических проявлений, а не в условиях острого кровотечения. В связи с этим важна ранняя диагностика ангиомиолипом.

\section{Рекомендации}

1. Пациентов нужно информировать о необходимости обратиться за неотложной медицинской помощью при появлении признаков кровотечения из ангиомиолипомы. (Сила доказательств: В. Качество доказательств: низкое. Польза: значительная. Согласие экспертов: очень хорошее).

2. Эмболизация должна быть методом выбора при кровотечении из ангиомиолипомы. В зависимости от местного опыта также могут использоваться нефрон-сберегающие хирургические вмешательства. (Сила доказательств: мнение экспертов / В. Качество доказательств: мнение экспертов. Польза: значительная. Согласие экспертов: очень хорошее).

3. В отдельных случаях, когда нельзя исключить злокачественную опухоль и показаны нефрон-сберегающие хирургические вмешательства, до их проведения следует выполнить эмболизацию. При этом необходимо учитывать технические аспекты, определяемые особенностями кровоснабжения опухоли и местным опытом. (Сила доказательств: С. Качество доказательств: низкое. Польза: умеренная. Согласие экспертов: очень хорошее).

\section{В каких случаях при отсутствии симптомов показано лечение ангиомиолипомы?}

Риск кровотечения определяется размерами ангиомиолипомы и высок при размерах опухоли $\geq 4 \mathrm{~cm}$ и наличии аневризм $\geq 5$ мм [50, 100, 101].

\section{Рекомендации}

1. Бессимптомные ангиомиолипомы почек размером $<4$ см не следует лечить, но нужно наблюдать с помощью ультразвука ежегодно до появления клинических проявлений. Если ультразвуковая диагностика недостаточно надежна по техническим причинам, следует выполнять КТ или МРТ (Сила доказательств: В. Качество доказательств: низкое. Польза: значительная. Согласие экспертов: очень хорошее).

2. Ангиомиолипомы почек размером > 4 см или с аневризмами $>5$ мм в диаметре имеют повышенный риск кровотечения и должны контролироваться ультразвуком 2 раза в год для оценки скорости роста. Следует рассматривать возможность их эмболизации и нефрон-сберегающих операций. (Сила доказательств: мнение экспертов / А. Качество доказательств: мнение экспертов. Польза: значительная. Согласие экспертов: очень хорошее).

\section{Трансплантация легких при ЛАМ}

На ЛАМ приходится 1,1 \% всех трансплантаций легких [102]. ЛАМ более благоприятен для трансплантации легких по сравнению с другими заболеваниями [38, 88, 103, 104]. В одном недавнем исследовании выживаемость пациентов после трансплантации легких по поводу ЛАМ составила $86 \%$ через 1 год, $76 \%$ через 3 года и $65 \%$ через 5 лет [104].

\section{Показания к трансплантации легких}

В связи с небольшим количеством больных и разной скоростью снижения легочной функции при ЛАМ однозначных рекомендаций не существует. В недавнем обзоре, посвященном больным ЛАМ, перенесшим трансплантацию легких, большинство имели тяжелую бронхиальную обструкцию и были оперированы при ОФВ ${ }_{1} \sim 25 \%$ и DLCO $-27 \%$ от должных величин [104]. Пациентов следует направлять на трансплантацию легких, когда они достигают III-IV функционального класса по Нью-Йоркской классификации (NYHA) с тяжелым нарушением функции легких и низкой переносимостью нагрузки ( $\mathrm{V}^{\prime} \mathrm{O}_{2 \max }<$ $50 \%$ долж., гипоксемия в покое). У пациентов старше 65 лет трансплантацию производят в исключительных случаях.

\section{Рекомендации}

Пациентов следует направлять на трансплантацию легких, когда они достигают III-IV функционального класса по NYHA с гипоксемией в покое, тяжелым нарушением функции легких и низкой переносимостью физической нагрузки ( $\mathrm{V}^{\prime} \mathrm{O}_{2 \max }<50 \%$ долж.). (Сила доказательств: А. Качество доказательств: среднее. Польза: значительная. Согласие экспертов: очень хорошее). 


\section{Какой тип трансплантации показан при ЛАМ?}

При ЛАМ выполняют пересадку как одного, так и обоих легких (чаще). После 2-сторонней трансплантации более значительно улучшается функция легких и реже развиваются осложнения ЛАМ, однако выживаемость пациентов при обоих типах трансплантации одинакова [104, 105].

\section{Рекомендации}

Выбор 1- или 2-сторонней трансплантации легких при ЛАМ определяется факторами хирургической техники и доступностью донорских органов. (Сила доказательств: В. Качество доказательств: среднее. Польза: умеренная. Согласие экспертов: очень хорошее).

\section{Особенности трансплантации легких при ТС}

Пациентам с ТС проводилась успешная пересадка легких по поводу тяжелого ЛАМ. [106, 107]. Трансплантация при ТС не имеет специфичных проблем, но у пациентов с ТС-ЛАМ обычно присутствует больше сопутствующих заболеваний, чем у пациентов со спорадическим ЛАМ. Влияние сопутствующей патологи и ее лечения требуют тщательной предтрансплантационной оценки.

\section{Рекомендации}

1. Наличие ТС не препятствует проведению трансплантации легких по поводу ЛАМ. У некоторых пациентов могут быть медицинские или когнитивные проблемы, связанные с ТС, которые мешают трансплантации. (Сила доказательств: В. Качество доказательств: низкое. Польза: значительная. Согласие экспертов: очень хорошее).

2. Пациенты с ТС-ЛАМ при решении вопроса о трансплантации легких нуждаются в тщательном всестороннем обследовании. (Сила доказательств: В. Качество доказательств: низкое. Польза: значительная. Согласие экспертов: очень хорошее).

\section{Влияет ли наличие ангиомиолипомы на совместимость трансплантата легкого?}

При ЛАМ важно выявить почечную ангиомиолипому до проведения трансплантации, поскольку доказана высокая вероятность кровотечения из крупных ангиомиолипом. В ходе предоперационного обследования ангиомиолипомы почек выявляют у $35-38$ \% пациентов с ЛАМ [107, 108]. Наличие ангиомиолипомы не повышает риска развития почечной недостаточности после трансплантации легких $[107,108]$.

\section{Рекомендации}

1. Ангиомиолипома не является противопоказанием для трансплантации легких, но может влиять на совместимость трансплантата, ход операции и послеоперационное течение. (Сила доказательств: В. Качество доказательств: низкое. Поль- за: значительная. Согласие экспертов: очень хорошее).

2. Наличие ангиомиолипомы почек должно быть установлено до операции, и угроза кровотечения должна быть устранена до трансплантации. (Сила доказательств: В. Качество доказательств: низкое. Польза: значительная. Согласие экспертов: очень хорошее).

\section{Следует ли оценивать вероятность рецидива ЛАМ в трансплантированном легком?}

Рецидив ЛАМ в пересаженном легком после 1- или 2 -сторонней трансплантации происходит редко и обычно не имеет клинических проявлений. Рецидив ЛАМ выявляют либо на аутопсии, либо при биопсии легкого, выполненной по другому поводу. Рецидив ЛАМ не влияет на выживание после трансплантации [38, 88, 104, 109-111].

\section{Рекомендации}

Не следует обследовать всех больных после трансплантации легкого для выявления рецидива ЛАМ. (Сила доказательств: D. Качество доказательств: низкое. Польза: отсутствует. Согласие экспертов: очень хорошее).

\section{Режимы иммуносупрессии после трансплантации легких при ЛАМ}

Используют те же режимы иммуносупрессии, что и после трансплантации по поводу других заболеваний $[88,103,107,112]$. Показатель заболеваемости в результате длительной иммуносупрессии при ЛАМ аналогичен таковому при прочих заболеваниях [88].

\section{Рекомендации}

Посттрансплантационный режим при ЛАМ не имеет особенностей по сравнению с другими заболеваниями. (Сила доказательств: С. Качество доказательств: низкое. Польза: умеренная. Согласие экспертов: очень хорошее).

\section{Заключение}

Данный документ является 1-м международным клиническим руководством по ЛАМ - редкому мультисистемному заболеванию. Его создание потребовало участия многих специалистов из разных стран, главным образом, из Европы и США. Отсутствие достаточной доказательной базы при этом редком заболевании потребовало использования соглашений между экспертами, в результате чего рекомендации основаны на сочетании доказательств и мнения экспертов. В данном документе впервые предложены диагностические критерии ЛАМ, рекомендации по обследованию пациентов, скринингу групп риска по ЛАМ, обсуждаются вопросы прогноза и ведения больных. Главная цель этого документа - улучшить состояние больных благодаря ранней диагностике и оптимиза- 
ции ведения в странах с разным уровнем здравоохранения. Руководство будет регулярно пересматриваться, и в него будут включаться все новые сведения.

\section{Литература}

1. Johnson S. Lymphangioleiomyomatosis: clinical features, management and basic mechanisms. Thorax 1999; 54: 254-264.

2. Franz D.N., Brody A., Meyer C. et al. Mutational and radiographic analysis of pulmonary disease consistent with lymphangioleiomyomatosis and micronodular pneumocyte hyperplasia in women with tuberous sclerosis. Am. J. Respir. Crit. Care Med. 2001; 164: 661-668.

3. Urban T., Lazor R., Lacronique J. et al. Pulmonary lymphangioleiomyomatosis. A study of 69 patients. Groupe d'Etudes et de Recherche sur les Maladies "Orphelines" Pulmonaires (GERM''O'P). Medicine (Baltimore) 1999; 78: 321-337.

4. Johnson S.R., Tattersfield A.E. Decline in lung function in lymphangioleiomyomatosis: relation to menopause and progesterone treatment. Am. J. Respir. Crit. Care Med. 1999; 160: 628-633.

5. Aubry M.C., Myers J.L., Ryu J.H. et al. Pulmonary lymphangioleiomyomatosis in a man. Am. J. Respir. Crit. Care Med. 2000; 162: 749-752.

6. Schiavina M., Di Scioscio V., Contini P. et al. Pulmonary lymphangioleiomyomatosis in a karyotypically normal man without tuberous sclerosis complex. Am. J. Respir. Crit. Care Med. 2007; 176: 96-98.

7. Avila N.A., Kelly J.A., Chu S.C. et al. Lymphangioleiomyomatosis: abdominopelvic CT and US findings. Radiology 2000; 216: 147-153.

8. Chu S.C., Horiba K., Usuki J. et al. Comprehensive evaluation of 35 patients with lymphangioleiomyomatosis. Chest 1999; 115: 1041-1052.

9. Moss J., DeCastro R., Patronas N.J. et al. Meningiomas in lymphangioleiomyomatosis. J. A. M. A. 2001; 286: 1879-1881.

10. Johnson S.R., Tattersfield A.E. Clinical experience of lymphangioleiomyomatosis in the UK. Thorax 2000; 55: 1052-1057.

11. Ryu J.H., Doerr C.H., Fisher S.D. et al. Chylothorax in lymphangioleiomyomatosis. Chest 2003; 123: 623-627.

12. Goncharova E.A., Goncharov D.A., Eszterhas A. et al. Tuberin regulates p70 S6 kinase activation and ribosomal protein S6 phosphorylation. A role for the TSC2 tumor suppressor gene in pulmonary lymphangioleiomyomatosis (LAM). J. Biol. Chem. 2002; 277: 30958-30967.

13. Kenerson H., Folpe A.L., Takayama T.K. et al. Activation of the mTOR pathway in sporadic angiomyolipomas and other perivascular epithelioid cell neoplasms. Hum. Pathol. 2007; 38: $1361-1371$.

14. Bissler J.J., McCormack F.X., Young L.R. et al. Sirolimus for angiomyolipoma in tuberous sclerosis complex or lymphangioleiomyomatosis. N. Engl. J. Med. 2008; 358: 140-151.

15. Davies D.M., Johnson S.R., Tattersfield A.E. et al. Sirolimus therapy in tuberous sclerosis or sporadic lymphangioleiomyomatosis. N. Engl. J. Med. 2008; 358: 200-203.

16. McCrory D.C., Lewis S.Z. Methodology and grading for pulmonary hypertension evidence review and guideline development. Chest 2004; 126 (Suppl. 1): 11S-13S.

17. Baumann M.H., Strange C., Heffner J.E. et al. Management of spontaneous pneumothorax: An American College of Chest Physicians Delphi Consensus Statement. Chest 2001; 119: 590-602.
18. Ryu J.H., Moss J., Beck G.J. et al. The NHLBI lymphangioleiomyomatosis registry: characteristics of 230 patients at enrollment. Am. J. Respir. Crit. Care Med. 2006; 173: 105-111.

19. Johnson S.R., Whale C.I., Hubbard R.B. et al. Survival and disease progression in UK patients with lymphangioleiomyomatosis. Thorax 2004; 59: 800-803.

20. Kitaichi M., Nishimura K., Itoh H. et al. Pulmonary lymphangioleiomyomatosis: a report of 46 patients including a clinicopathologic study of prognostic factors. Am. J. Respir. Crit. Care Med. 1995; 151: 527-533.

21. Taveira-DaSilva A.M., Stylianou M.P., Hedin C.J. et al. Decline in lung function in patients with lymphangioleiomyomatosis treated with or without progesterone. Chest 2004; 126: 1867-1874.

22. Carrington C.B., Cugell D.W., Gaensler E.A. et al. Lymphangioleiomyomatosis. Physiologic-pathologic-radiologic correlations. Am. Rev. Respir. Dis. 1977; 116: 977-995.

23. Matsui K., Beasley M.B., Nelson W.K. et al. Prognostic significance of pulmonary lymphangioleiomyomatosis histologic score. Am. J. Surg. Pathol. 2001; 25: 479-484.

24. Corrin B., Liebow A., Friedman P. Pulmonary lymphangiomyomatosis. A review. Am. J. Pathol. 1975; 79: 348-382.

25. Bonetti F., Chiodera P.L., Pea M. et al. Transbronchial biopsy in lymphangiomyomatosis of the lung. HMB45 for diagnosis. Am. J. Surg. Pathol. 1993; 17: 1092-1102.

26. Matsui K., Takeda K., Yu Z.X. et al. Downregulation of estrogen and progesterone receptors in the abnormal smooth muscle cells in pulmonary lymphangioleiomyomatosis following therapy. An immunohistochemical study. Am. J. Respir. Crit. Care Med. 2000; 161: 1002-1009.

27. Glassberg M.K., Elliot S.J., Fritz J. et al. Activation of the estrogen receptor contributes to the progression of pulmonary lymphangioleiomyomatosis via MMP-induced cell invasiveness. J. Clin. Endocrinol. 2008; 93: 1625-1633.

28. Zompatori M., Poletti V., Battista G. et al. Diffuse cystic lung disease in the adult patient. Radiol. Med. (Torino) 2000; 99: $12-21$.

29. Steagall W.K., Glasgow C.G., Hathaway O.M. et al. Genetic and morphologic determinants of pneumothorax in lymphangioleiomyomatosis. Am. J. Physiol. Lung Cell Mol. Physiol. 2007; 293: L800-L808.

30. Avila N.A., Chen C.C., Chu S.C. et al. Pulmonary lymphangioleiomyomatosis: correlation of ventilation-perfusion scintigraphy, chest radiography, and CT with pulmonary function tests. Radiology 2000; 214: 441-446.

31. Lenoir S., Grenier P., Brauner M.W. et al. Pulmonary lymphangiomyomatosis and tuberous sclerosis: comparison of radiographic and thin-section CT findings. Radiology 1990; 175: 329-334.

32. Muller N.L., Chiles C., Kullnig P. Pulmonary lymphangiomyomatosis: correlation of CT with radiographic and functional findings. Radiology 1990; 175: 335-339.

33. Abbott G.F., Rosado-de-Christenson M.L., Frazier A.A. et al. From the archives of the AFIP: lymphangioleiomyomatosis: radiologicpathologic correlation. Radiographics 2005; 25: 803-828.

34. Helenon O., Merran S., Paraf F. et al. Unusual fat-containing tumors of the kidney: a diagnostic dilemma. Radiographics 1997; 17: 129-144.

35. Roach E.S., Sparagana S.P. Diagnosis of tuberous sclerosis complex. J. Child Neurol. 2004; 19: 643-649.

36. Taveira-DaSilva A.M., Hedin C., Stylianou M.P. et al. Reversible airflow obstruction, proliferation of abnormal 
smooth muscle cells, and impairment of gas exchange as predictors of outcome in lymphangioleiomyomatosis. Am. J. Respir. Crit. Care Med. 2001; 164: 1072-1076.

37. Taylor J.R., Ryu J., Colby T.V. et al. Lymphangioleiomyomatosis. Clinical course in 32 patients. N. Engl. J. Med. 1990; 323: 1254-1260.

38. Boehler A., Speich R., Russi E.W. et al. Lung transplantation for lymphangioleiomyomatosis. N. Engl. J. Med. 1996; 335: $1275-1280$.

39. Taveira-DaSilva A.M., Stylianou M.P., Hedin C.J. et al. Maximal oxygen uptake and severity of disease in lymphangioleiomyomatosis. Am. J. Respir. Crit. Care Med. 2003; 168: $1427-1431$.

40. ATS Statement: Guidelines for the six-minute walk test. Am. J. Respir. Crit. Care Med. 2002; 166: 111-117.

41. Costello L.C., Hartman T.E., Ryu J.H. High frequency of pulmonary lymphangioleiomyomatosis in women with tuberous sclerosis complex. Mayo Clin. Proc. 2000; 75: 591-594.

42. Moss J., Avila N.A., Barnes P.M. et al. Prevalence and clinical characteristics of lymphangioleiomyomatosis (LAM) in patients with tuberous sclerosis complex. Am. J. Respir. Crit. Care Med. 2001; 164: 669-671.

43. Castro M., Shepherd C.W., Gomez M.R. et al. Pulmonary tuberous sclerosis. Chest 1995; 107: 189-195.

44. Kim N.R., Chung M.P., Park C.K. et al. Pulmonary lymphangioleiomyomatosis and multiple hepatic angiomyolipomas in a man. Pathol. Int. 2003; 53: 231-235.

45. Casper K.A., Donnelly L.F., Chen B. et al. Tuberous sclerosis complex: renal imaging findings. Radiology 2002; 225: 451-456.

46. Ewalt D.H., Sheffield E., Sparagana S.P. et al. Renal lesion growth in children with tuberous sclerosis complex. J. Urol. (Baltimore) 1998; 160: 141-145.

47. Weiner D.M., Ewalt D.H., Roach E.S. et al. The tuberous sclerosis complex: a comprehensive review. J. Am. Coll. Surg. 1998; 187: 548-561.

48. L'Hostis H., Deminiere C., Ferriere J.M. et al. Renal angiomyolipoma: a clinicopathologic, immunohistochemical, and follow-up study of 46 cases. Am. J. Surg. Pathol. 1999; 23: 1011-1020.

49. Nelson C.P., Sanda M.G. Contemporary diagnosis and management of renal angiomyolipoma. J. Urol. (Baltimore) 2002; 168: 1315-1325.

50. Steiner M.S., Goldman S.M., Fishman E.K. et al. The natural history of renal angiomyolipoma. J. Urol. (Baltimore) 1993; 150: 1782-1786.

51. Kennelly M.J., Grossman H.B., Cho K.J. Outcome analysis of 42 cases of renal angiomyolipoma. J. Urol. (Baltimore) 1994; 152: 1988-1991.

52. Dickinson M., Ruckle H., Beaghler M. et al. Renal angiomyolipoma: optimal treatment based on size and symptoms. Clin. Nephrol. 1998; 49: 281-286.

53. Lazor R., Valeyre D., Lacronique J. et al. Low initial KCO predicts rapid $\mathrm{FEV}_{1}$ decline in pulmonary lymphangioleiomyomatosis. Respir. Med. 2004; 98: 536-541.

54. Cohen M.M., Pollock-BarZiv S., Johnson S.R. Emerging clinical picture of lymphangioleiomyomatosis. Thorax 2005; 60: 875-879.

55. Almoosa K.F., Ryu J.H., Mendez J. et al. Management of pneumothorax in lymphangioleiomyomatosis: effects on recurrence and lung transplantation complications. Chest 2006; 129: 1274-1281.

56. Fujimoto M., Ohara N., Sasaki H. et al. Pregnancy complicated with pulmonary lymphangioleiomyomatosis: case report. Clin. Exp. Obstetr. Gynecol. 2005; 32: 199-200.
57. Brunelli A., Catalini G., Fianchini A. Pregnancy exacerbating unsuspected mediastinal lymphangioleiomyomatosis and chylothorax. Int. J. Gynaecol. Obstetr. 1996; 52: 289-290.

58. McLoughlin L., Thomas G., Hasan K. Pregnancy and lymphangioleiomyomatosis: anaesthetic management. Int. J. Obstetr. Anesth. 2003; 12: 40-44.

59. Yockey C.C., Riepe R.E., Ryan K. Pulmonary lymphangioleiomyomatosis complicated by pregnancy. Kans. Med. 1986; 87: 277-278, 293.

60. Warren S.E., Lee D., Martin V. et al. Pulmonary lymphangiomyomatosis causing bilateral pneumothorax during pregnancy. Ann. Thorac. Surg. 1000; 55: 998-1000.

61. Cohen M.M., Freyer A.M., Johnson S.R. Pregnancy experiences among women with lymphangioleiomyomatosis. Respir. Med. 2009; 103: 766-772.

62. Raft J., Lalot J.M., Meistelman C. et al. Renal angiomyolipoma rupture during pregnancy. Gynecol. Obstetr. Fertil 2006; 34: 917-919.

63. Storm D.W., Mowad J.J. Conservative management of a bleeding renal angiomyolipoma in pregnancy. Obstetr. and Gynecol. 2006; 107: 490-492.

64. Morales J.P., Georganas M., Khan M.S. et al. Embolization of a bleeding renal angiomyolipoma in pregnancy: case report and review. Cardiovasc. Intervent. Radiol. 2005; 28: 265-268.

65. Mascarenhas R., McLaughlin P. Haemorrhage from angiomyolipoma of kidney during pregnancy - a diagnostic dilemma. Ir. Med. J. 2001; 94: 83-84.

66. Yano $S$. Exacerbation of pulmonary lymphangioleiomyomatosis by exogenous oestrogen used for infertility treatment. Thorax 2002; 57: 1085-1086.

67. Shen A., Iseman M.D., Waldron J.A. et al. Exacerbation of pulmonary lymphangioleiomyomatosis by exogenous estrogens. Chest 1987; 91: 782-785.

68. Wilson A.M., Slack H.L., Soosay S.A. et al. Lymphangioleiomyomatosis. A series of three case reports illustrating the link with high oestrogen states. Scott. Med. J. 2001; 46: $150-152$.

69. Oberstein E.M., Fleming L.E., Gomez-Marin O. et al. Pulmonary lymphangioleiomyomatosis (LAM): examining oral contraceptive pills and the onset of disease. J. Women's Hlth 2003; 12: 81-85.

70. Pollock-BarZiv S., Cohen M.M., Downey G.P. et al. Air travel in women with lymphangioleiomyomatosis. Thorax 2007; 62: $176-180$.

71. Rehabilitation BTSSoCs-coP. BTS statement on pulmonary rehabilitation. Thorax 2001; 56: 827-834.

72. Managing stable COPD. Thorax 2004; 59: (Suppl. 1), i39-i130.

73. Taveira-DaSilva A.M., Stylianou M.P., Hedin C.J. et al. Bone mineral density in lymphangioleiomyomatosis. Am. J. Respir. Crit. Care Med. 2005; 171: 61-67.

74. Adamson D., Heinrichs W.L., Raybin D.M. et al. Successful treatment of pulmonary lymphangiomyomatosis with oophorectomy and progesterone. Am. Rev. Respir. Dis. 1985; 132: 916-921.

75. Anker N., Francis D., Viskum K. 2 cases of lymphangioleiomyomatosis treated by hormonal manipulation. Ugeskr. Laeg. 1993; 155: 2354-2356.

76. Banner A.S., Carrington C.B., Emory W.B. et al. Efficacy of oophorectomy in lymphangioleiomyomatosis and benign metastasizing leiomyoma. N. Engl. J. Med. 1981; 305: 204-209.

77. Itoi K., Kuwabara M., Okubo K. et al. A case of pulmonary lymphangiomyomatosis treated with bilateral oophorecto- 
my and methyl-progesterone-acetate. Nihon Kyobu Shikkan Gakkai Zasshi 1993; 31: 1146-1150.

78. Kanbe A., Hajiro K., Adachi Y. et al. Lymphangiomyomatosis associated with chylous ascites and high serum CA125 levels: a case report. Jpn J. Med. 1987; 26: 237-242.

79. Brock E.T., Votto J.J. Lymphangioleiomyomatosis: treatment with hormonal manipulation. N. Y. St. J. Med. 1986; 86: 533-536.

80. Klein M., Krieger O., Ruckser R. et al. Treatment of lymphangioleiomyomatosis by ovariectomy, interferon alpha $2 \mathrm{~b}$ and tamoxifen - a case report. Arch. Gynecol. Obstetr. 1992; 252: 99-102.

81. Svendsen T.L., Viskum K., Hansborg N. et al. Pulmonary lymphangioleiomyomatosis: a case of progesterone receptor positive lymphangioleiomyomatosis treated with medroxyprogesterone, oophorectomy and tamoxifen. Br. J. Dis. Chest 1984; 78: 264-271.

82. Tomasian A., Greenberg M.S., Rumerman H. Tamoxifen for lymphangioleiomyomatosis. N. Engl. J. Med. 1982; 306: $745-746$.

83. de la Fuente J., Paramo C., Roman F. et al. Lymphangioleiomyomatosis: unsuccessful treatment with luteinizinghormone-releasing hormone analogues. Eur. J. Med. 1993; 2: $377-378$.

84 . Desurmont S., Bauters C., Copin M.C. et al. Treatment of pulmonary lymphangioleiomyomatosis using a GnRH agonist. Rev. Mal. Respir. 1996; 13: 300-304.

85. Rossi G.A., Balbi B., Oddera S. et al. Response to treatment with an analog of the luteinizing-hormone-releasing hormone in a patient with pulmonary lymphangioleiomyomatosis. Am. Rev. Respir. Dis. 1991; 143: 174-176.

86. Harari S., Cassandro R., Chiodini J. et al. Effect of a gonadotrophin-releasing hormone analogue on lung function in lymphangioleiomyomatosis. Chest 2008; 133: 448-454.

87. Detterbeck F.C., Egan T.M., Mill M.R. Lung transplantation after previous thoracic surgical procedures. Ann. Thorac. Surg. 1995; 60: 139-143.

88. Pechet T.T., Meyers B.F., Guthrie T.J. et al. Lung transplantation for lymphangioleiomyomatosis. J. Heart Lung Transplant. 2004; 23: 301-308.

89. Morimoto N., Hirasaki S., Kamei T. et al. Pulmonary lymphangiomyomatosis (LAM) developing chylothorax. Intern. Med. 2000; 39: 738-741.

90. Christodoulou M., Ris H.-B., Pezzetta E. Video-assisted right supradiaphragmatic thoracic duct ligation for non-traumatic recurrent chylothorax. Eur. J. Cardiothorac. Surg. 2006; 29: $810-814$

91. Avila N.A., Dwyer A.J., Rabel A. et al. CT of pleural abnormalities in lymphangioleiomyomatosis and comparison of pleural findings after different types of pleurodesis. Am. J. Roentgenol. 2006; 186: 1007-1012.

92. Druelle S., Aubry P., Levi-Valensi P. Pulmonary lymphangiomyomatosis: a 3-year follow-up of medroxyprogesterone acetate therapy. Apropos of a case. Rev. Pneumol. Clin. 1995; 51: 284-287.

93. Zanella A., Toppan P., Nitti D. et al. Pulmonary lymphangioleiomyomatosis: a case report in postmenopausal woman treated with pleurodesis and progesterone (medroxyprogesterone acetate). Tumori 1996; 82: 96-98.

94. Worjcik P., Otto T., Jagieiio $R$. et al. Use of pleuro-peritoneal shunt in the treatment of chronic chylothorax. Pneumonol. Alergol. Pol. 1998; 66: 473-479
95. Calvo E., Amarillas L., Mateos M.A. et al. Lymphangioleiomyomatosis, chylous ascites, and diet. Dig. Dis. Sci. 1996; 41: 591-593.

96. Hamlin J.A., Smith D.C., Taylor F.C. et al. Renal angiomyolipomas: long-term follow-up of embolization for acute hemorrhage. Can. Assoc. Radiol. J. 1997; 48: 191-198.

97. Williams J.M., Racadio J.M., Johnson N.D. et al. Embolization of renal angiomyolipomata in patients with tuberous sclerosis complex. Am. J. Kidney Dis. 2006; 47: 95-102.

98. Igarashi A., Masuyama T., Watanabe K. et al. Long-term result of the transcatheter arterial embolization for ruptured renal angiomyolipoma. Nippon Hinyokika Gakkai Zasshi 2002; 93: 702-706.

99. Bender B., Yunis E. The pathology of tuberous sclerosis. Pathol. Annu. 1982; 17: 339-382.

100. van Baal J.G., Smits N.J., Keeman J.N. et al. The evolution of renal angiomyolipomas in patients with tuberous sclerosis. J. Urol. (Baltimore) 1994; 152: 35-38.

101. Yamakado K., Tanaka N., Nakagawa T. et al. Renal angiomyolipoma: relationships between tumor size, aneurysm formation, and rupture. Radiology 2002; 225: $78-82$.

102. Trulock E.P. Lung transplantation: special considerations and outcome in LAM. In: Moss J., ed. LAM and other diseases characterised by smooth muscle proliferation. New York: Marcel Dekker; 1999. 65-78.

103. Pigula F.A., Griffith B.P., Zenati M.A. et al. Lung transplantation for respiratory failure resulting from systemic disease. Ann. Thorac. Surg. 1997; 64: 1630-1634.

104. Kpodonu J., Massad M.G., Chaer R.A. et al. The US experience with lung transplantation for pulmonary lymphangioleiomyomatosis. J. Heart Lung Transplant. 2005; 24: 1247-1253.

105. Boehler A. Lung transplantation for cystic lung diseases: lymphangioleiomyomatosis, histiocytosis $\mathrm{x}$, and sarcoidosis. Semin. Respir. Crit. Care Med. 2001; 22: 509-516.

106. Benden C., Rea F., Behr J. et al. Lung transplantation for lymphangioleiomyomatosis: the European experience. J. Heart Lung Transplant. 2009; 28: 1-7.

107. Reynaud-Gaubert M., Mornex J.F., Mal H. et al. Lung transplantation for lymphangioleiomyomatosis: the French experience. Transplantation 2008; 86: 515-520.

108. Collins J., Muller N.L., Kazerooni E.A. et al. Lung transplantation for lymphangioleiomyomatosis: role of imaging in the assessment of complications related to the underlying disease. Radiology 1999; 210: 325-332.

109. Nine J.S., Yousem S.A., Paradis I.L. et al. Lymphangioleiomyomatosis: recurrence after lung transplantation. J. Heart Lung Transplant. 1994; 13: 714-719.

110. Bittmann I., Rolf B., Amann G. et al. Recurrence of lymphangioleiomyomatosis after single lung transplantation: New insights into pathogenesis. Hum. Pathol. 2003; 34: 95-98.

111. Karbowniczek M., Astrinidis A., Balsara B.R. et al. Recurrent lymphangiomyomatosis after transplantation: genetic analyses reveal a metastatic mechanism. Am. J. Respir. Crit. Care Med. 2003; 167: 976-982.

112. Benden C., Boehler A., Faro A. Pediatric lung transplantation: Literature review. Pediatr. Transplant. 2008; 12: 266-273.

Перевод И.Э.Степаняна, С.И.Чикиной 